\title{
Inflatonless inflation
}

\author{
A. Dobado and A. L. Maroto \\ Departamento de Física Teórica, Universidad Complutense de Madrid, 28040 Madrid, Spain
}

(Received 9 August 1994; revised manuscript received 3 February 1995)

\begin{abstract}
Whichever turns out to be the real theory of gravitation, the corresponding low-energy effective Lagrangian will probably contain higher derivative terms. In this work we study the general conditions on those terms in order to produce enough inflation to solve some of the problems of the standard Friedmann-Robertson-Walker cosmology in the absence of any inflaton field. We apply our results to some particular scenarios where higher derivative terms appear in the effective Lagrangian for gravity, such as those coming from graviton (two) loops or integrating out ordinary matter (such as the one present in the standard model).
\end{abstract}

PACS number(s): 98.80.Cq, 04.50.+h

\section{INTRODUCTION}

In recent years, the general physical idea of a period of enormous inflation in the early history of our universe has emerged as one of the most simple and successful ways to solve many of the problems of the FriedmannRobertson-Walker cosmological scenario [1,2]. The list of these problems includes flatness, the horizon problem, and the origin of the density fluctuations needed to produce the current galactic structure.

Concerning the nature of the physical mechanism responsible for inflation there are many of them proposed in the literature. However, none of them has been universally accepted. Typically they include the action of some scalar field called the inflaton taking some vacuum expectation value. The corresponding energy density plays the role of an effective cosmological constant which gives rise to a nearly de Sitter phase of exponential expansion. The concrete nature of the inflaton field depends on the different microphysics models considered.

In this paper we deal with another kind of approach based on the possibility of having inflation without any inflaton field. This possibility was envisaged by Starobinsky some time ago [3]. Even before the inflationary paradigm was established, this author discovered that the addition of some terms to the Einstein equation of motion gives rise to de Sitter spaces as solutions. In fact those terms can be obtained in the effective action for gravity as the result of integrating out conformal free matter fields. The possibilities for the Starobinsky mechanism to produce successful inflation were studied by Starobinsky himself and by Vilenkin in [3,4]. More recently it was realized by the authors [5] that the standard EinsteinHilbert action supplemented with a six derivative term gives rise to a modified equation of motion supporting de Sitter solutions. Moreover, the introduction of this term is not arbitrary at all but is necessary for the renormalizability of the effective low-energy theory of gravitation at the two-loop level [6].

Whatever turns out to be the most fundamental theory of gravitation (such as superstrings or any other), it is clear that at low energy it must lead to the standard
Einstein-Hilbert Lagrangian proportional to the scalar curvature $R$ which has two derivatives of the metric $g_{\alpha \beta}$ and therefore leads to graviton scattering amplitudes of the order of the external momenta over the Planck mass $M_{P}$ squared.

This is a good approximation at low energies, but when higher energies are considered, higher derivative terms will in general appear in the effective gravitational Lagrangian. These terms will be affected by adimensional constants and the necessary $M_{P}$ factors depending on the dimension of the operator. The adimensional constants play a double role: first they carry the information about the underlying theory of gravitation; second they will absorb the divergences that appear when quantum corrections; i.e., loops are computed with the effective Lagrangian. These loops can contain matter fields, like in the Starobinsky case, or gravitons (together with the corresponding ghosts) as happens in the model considered in [5], or whatever.

This scheme has many analogies with the phenomenological Lagrangian approach proposed by Weinberg [7] for the description of the low-energy hadron interactions and further elaborated by Gasser and Leutwyler [8], i.e., the so-called chiral perturbation theory $(\chi \mathrm{PT})$. This technique has also been applied to the parametrization of the scattering amplitudes of the longitudinal components of the electroweak bosons at the TeV scale [9]. More recently $[5,10]$ the same philosophy has been proposed to describe the low-energy action for gravity [11].

At this point a natural question arises: which are the new higher derivative terms that one must include in the low-energy effective pure gravity Lagrangian? In principle, any general covariant combination of scalar curvature and the Ricci and Riemann tensors $R, R_{\alpha \beta}$, and $R_{\alpha \beta \gamma \delta}$ should be included. For example, one has three possible independent four derivative terms that can be written as $R^{2}, R^{\alpha \beta} R_{\alpha \beta}$, and $-4 R^{\alpha \beta}{ }_{\gamma \delta} R^{\gamma \delta}{ }_{\alpha \beta}+16 R^{\alpha \beta} R_{\alpha \beta}-4 R^{2}$. The third term is a total derivative related with the Euler class of the space-time manifold.

In the general case we will assume the action for gravity to be the space-time integral of an arbitrary local analytical scalar function of the scalar curvature and the Ricci 
and Riemann tensors added to the standard EinsteinHilbert action or, in other words,

$$
S_{G}=\int d^{4} x \sqrt{-g}\left(-\frac{M_{P}^{2}}{16 \pi} R+F\left(R, R_{\alpha \beta}, R_{\alpha \beta \gamma \delta}\right)\right) .
$$

Note however that, in principle, the effective action for gravity could depend also on higher covariant derivatives of the Riemann and Ricci tensors and the curvature scalar and contain nonlocal terms but these possibilities will not be considered here.

As discussed above, in this work we are interested in studying the possibility of having inflation without the introduction of any inflaton field. Therefore we can start studying the precise conditions to be required on the function $F$ appearing in the action in Eq. (1) leading to de Sitter space-times as solutions of the corresponding equation of motion. Of course, it is clear that this is not the only possibility for having inflation since it should be enough with the much more general condition $\dot{H}>-H^{2}$ (with $H$ being the Hubble parameter). However, to start with, we will consider first this kind of simple solution and we will discuss the general case at the end of Sec. III.

The physical meaning of these nonperturbative solutions (such as the de Sitter solution) which can eventually appear in the modified Einstein-Hilbert action but not in the Einstein-Hilbert action itself (for null cosmological contant) has been questioned in the literature. For instance, in [12] it is argued that these solutions do not appear in a correct perturbative treatment. However, we think that this is not necessarily the case since there are other similar scenarios where these nonperturbative solutions have a well-defined physical meaning. This is the case, for instance, of the above-mentioned $\chi \mathrm{PT}$. There, the higher derivative terms added to the two derivative one (which would be analogous to the EinsteinHilbert Lagrangian) not only modify in a perturbative way the pion scattering amplitudes; in addition, they give rise to other well-known nonperturbative solutions called skyrmions which cannot be obtained with the two derivative term alone. Moreover, the skyrmion has been shown to provide a phenomenologically consistent description of baryons in the celebrated paper by Adkinds, Nappi, and Witten [13]. Therefore we understand that the physical meaning of the possible nonperturbative solutions appearing when higher derivative terms are added to the Einstein-Hilbert action should not be a priori discarded.

The possibility of having de Sitter solutions coming from generalizations of the Einstein-Hilbert action which are analytical functions on the scalar curvature has been considered by Barrow and Ottewill [14] who have studied the existence and stability of homogeneous and isotropic cosmological solutions in this case. On that score our work will follow this approach extending it for arbitrary analytical functions on the Riemann and Ricci tensors and the scalar curvature. On the other hand, it is well known [15] that some of these higher derivative models can be related to the usual inflaton ones via conformal transformations. Specifically, gravity Lagrangians which are arbitrary functions of the scalar curvature are conformally related to that of Einstein's gravity plus a scalar field whose self-interactions depend on the specific form of the higher order terms. However, this is not the case when the modified gravity Lagrangian is an arbitrary function of the Riemann and Ricci tensor and the scalar curvature as we are considering here. In this case it is also possible to relate it to Einstein's gravity plus matter fields, but the matter sector includes not only scalar but also tensor fields [16].

\section{EXISTENCE OF (ANTI-)de SITTER SOLUTIONS}

For maximally symmetric space-times it is always possible to write the Riemann tensor just in terms of the scalar curvature:

$$
R_{\alpha \beta \gamma \delta}=\frac{1}{n(n-1)} R\left(g_{\alpha \gamma} g_{\beta \delta}-g_{\alpha \delta} g_{\beta \gamma}\right)
$$

where $n$ is the dimension of space-time. Therefore, we can rewrite the action integral in Eq. (1) in terms of the scalar curvature only:

$$
S_{G}=\int d^{4} x \sqrt{-g}\left(-\frac{M_{P}^{2}}{16 \pi} R+G(R)\right)
$$

for some well-defined analytical function $G$ to be obtained from the original $F$ function in Eq. (1).

The equation of motion corresponding to this action can be found to be

$$
\begin{aligned}
\frac{M_{P}^{2}}{16 \pi}\left(R_{\alpha \beta}-\frac{1}{2} g_{\alpha \beta} R\right)= & -\frac{1}{2} g_{\alpha \beta} G(R)+R_{\alpha \beta} G^{\prime}(R) \\
& +g_{\alpha \beta} \square G^{\prime}(R)-G^{\prime}(R)_{; \alpha \beta},
\end{aligned}
$$

where a prime denotes derivative respect to $R$. In order to find the condition for having (anti-)de Sitter spacetimes as solution of this equation it is important to remember that these are maximally symmetric space-times of constant scalar curvature $R$. Therefore, in this case the above equation of motion reduces to

$$
\frac{M_{P}^{2}}{16 \pi} R=2 G(R)-R G^{\prime}(R)
$$

which is the condition for having (anti-)de Sitter solutions. In principle, this equation can have zero, one, or several solutions. In the first case no de Sitter inflation is possible but in the other cases one or more de Sitter phases can be present. It is immediately seen from the equation above that the addition of quadratic terms to the Einstein-Hilbert action [i.e., $F=\alpha R^{2}+\beta R_{\mu \nu} R^{\mu \nu}+$ $\gamma R_{\alpha \beta \gamma \delta} R^{\alpha \beta \gamma \delta}$, or in other words $G(R) \propto R^{2}$ ] does not lead to any solutions different from $R=0$ which corresponds to the Minkowski space-time. This, of course, does not mean that those terms cannot lead to inflation, i.e., an expansion phase where $\dot{H}>-H^{2}$, but only that they cannot give rise to de Sitter inflationary solutions. 


\section{ROBERTSON-WALKER PERTURBATIONS}

Let us consider now the standard Robertson-Walker metric

$$
d \tau^{2}=d t^{2}-a^{2}(t)\left(\frac{d r^{2}}{1-k r^{2}}+r^{2} d \theta^{2}+r^{2} \sin ^{2} \theta d \phi^{2}\right)
$$

(we will follow the notation convention of Weinberg's book [17]), where $k=1,0,-1$ corresponds to a closed, flat, or open space and $a(t)$ is the universe scale parameter. For the sake of simplicity, in the following we will concentrate in the flat or $k=0$ universe. This is not in general a maximally symmetric space-time but it includes a particular case which indeed is, namely, $a(t)=a\left(t_{0}\right) \exp \left[H_{0}\left(t-t_{0}\right)\right]$. This fact makes it possible to use this metric to study small perturbations around the (anti-)de Sitter solutions found in Eq. (5) and, in turn, the perturbative analysis will provide an estimation of the duration of each inflationary de Sitter period as we will see later on. Proceeding in this way we first note that the Riemann tensor for this metric has only the following six nonvanishing independent components:

$$
\begin{gathered}
R_{t r}^{t r}=R_{t \theta}^{t \theta}=R_{t \phi}^{t \phi}=-\frac{\ddot{a}}{a}, \\
R_{\theta \phi}^{\theta \phi}=R_{r \theta}^{r \theta}=R_{r \phi}^{r \phi}=-\frac{\dot{a}^{2}}{a^{2}} .
\end{gathered}
$$

Since only these functions of $a$ and its derivatives will appear in the action it is then more useful in practice to work with them as new variables. Thus we define $b(t)=\ln [a(t)], H(t) \equiv \dot{b}(t)=\dot{a} / a$, and $\dot{H}(t)=\ddot{b}(t)=$ $\ddot{a} / a-\dot{a}^{2} / a^{2}$. In terms of the Hubble parameter $H(t)$ that we have just defined, the (anti-)de Sitter space-time corresponding to $a(t)=a\left(t_{0}\right) \exp \left[H_{0}\left(t-t_{0}\right)\right]$ is simply $H=H_{0}$ and the action integral in Eq. (1) reads

$$
S_{G} \propto \int d t e^{3 b} L(H, \dot{H}) .
$$

Here a global volume factor has been extracted and the function $L(H, \dot{H})$ which is obtained from the function $F$ in Eq. (1) includes the Einstein-Hilbert part of the Lagrangian. The equation of motion for the Hubble parameter $H$ obtained from the action above reads

$$
\begin{array}{r}
3 L-3 H \frac{\partial L}{\partial H}-\frac{d}{d t} \frac{\partial L}{\partial H}+3 \dot{H} \frac{\partial L}{\partial \dot{H}}+9 H^{2} \frac{\partial L}{\partial \dot{H}}+6 H \frac{d}{d t} \frac{\partial L}{\partial \dot{H}} \\
+\frac{d^{2}}{d t^{2}} \frac{\partial L}{\partial \dot{H}}=0 .
\end{array}
$$

This equation obviously possesses the solutions $H(t)=$ $H_{0}$ corresponding to the (anti-)de Sitter space-times found in Eq. (5). In order to study the stability of these solutions we must consider the behavior of small perturbation around them: that is,

$$
H(t)=H_{0}+\delta(t), \quad \dot{H}(t)=\dot{\delta}(t)
$$

Expanding $L(H, \dot{H})$ and its derivatives to the first order in $\delta$ we find

$$
\begin{gathered}
L=L_{0}+L_{1} \delta+L_{2} \dot{\delta}+O\left(\delta^{2}\right), \\
\frac{\partial L}{\partial H}=L_{1}+L_{11} \delta+L_{12} \dot{\delta}+O\left(\delta^{2}\right), \\
\frac{\partial L}{\partial \dot{H}}=L_{2}+L_{21} \delta+L_{22} \dot{\delta}+O\left(\delta^{2}\right),
\end{gathered}
$$

where we have defined the coefficients

$$
\begin{aligned}
L_{0} & =L\left(H_{0}, 0\right), \\
L_{1} & =\frac{\partial L}{\partial H}\left(H_{0}, 0\right), \\
L_{2} & =\frac{\partial L}{\partial \dot{H}}\left(H_{0}, 0\right), \\
L_{11} & =\frac{\partial^{2} L}{\partial H^{2}}\left(H_{0}, 0\right), \\
L_{12}=L_{21} & =\frac{\partial^{2} L}{\partial H \partial \dot{H}}\left(H_{0}, 0\right), \\
L_{22} & =\frac{\partial^{2} L}{\partial \dot{H}^{2}}\left(H_{0}, 0\right) .
\end{aligned}
$$

Finally, substituting these expansions in Eq. (10) and neglecting terms of second order we obtain the linearized equation for $\delta(t)$, which can be written as

$$
3\left(L_{0}-H_{0} L_{1}+3 H_{0}^{2} L_{2}\right)+\frac{1}{3 H_{0}} \frac{d}{d t} \mathcal{F}+\mathcal{F}=0
$$

where $\mathcal{F}$ stands for

$$
\mathcal{F}=L_{22} \ddot{\delta}+3 H_{0} L_{22} \dot{\delta}+\delta\left(6 L_{2}+3 H_{0} L_{12}-L_{11}\right) .
$$

Now the condition in Eq. (5) for having (anti-)de Sitter solutions can be rewritten as a condition on $H_{0}$

$$
L_{0}-H_{0} L_{1}+3 H_{0}^{2} L_{2}=0 .
$$

The resolution of the linearized equation for $\delta(t)$ [Eq. (16)] around each solution $H_{0}^{(i)}$ of Eq. (18) simply becomes the resolution of equation $\mathcal{F}=0$ whose solutions are any linear combination of modes $\exp t / \tau$ with

$$
\begin{aligned}
\frac{1}{\tau^{(i)}}= & -\frac{3 H_{0}^{(i)}}{2} \pm\left(\frac{9 H_{0}^{(i)^{2}}}{4}-\frac{1}{L_{22}^{(i)}}\left(6 L_{2}^{(i)}+3 H_{0}^{(i)} L_{12}^{(i)}\right.\right. \\
& \left.\left.-L_{11}^{(i)}\right)\right)^{(1 / 2)} .
\end{aligned}
$$

Here $i$ runs over the number of real solutions of Eq. (18). The stability of these inflationary solutions is given by the signs of $\tau^{(i)}$. When both are growing modes, i.e., $\tau^{(i)}>0$, the corresponding solution is unstable to any perturbation. If both modes are decreasing, i.e., $\tau^{(i)}<0$, the solution is stable and, as a consequence of that, the inflationary phase will be endless (at least if possible tunneling effects are neglected). Equation (19) guarantees that a stable mode is always present for de Sitter $H_{0}>0$ solutions. Therefore, the kind of physically interesting solutions are, in principle, those with one stable and one unstable mode. This is so because they assure that if the universe had started in this de 
Sitter phase it could have decayed into an acceptable solution at late times. On the other hand, in a more general case, if the initial conditions are arbitrary, at least there exists an area in the phase space where the solutions satisfy $\dot{H}>-H^{2}$, i.e., the criterion for the existence of inflationary solutions. In addition, the value of $\tau^{(i)}$ for the unstable mode gives an estimation of the duration of the corresponding de Sitter phase and, in turn, of the number of $e$ foldings $N_{e}^{(i)}$ produced during this period:

$$
N_{e}^{(i)} \equiv \int H(t) d t=H_{0}^{(i)} \tau^{(i)}
$$

In general this is a lower bound to the number of $e$ foldings produced during the whole inflationary era, since the end of the de Sitter phase does not mean the end of inflation. The duration of the whole inflationary phase depends, in the general case, on the initial conditions and the dynamics of the model. Therefore it should be estimated in detail in the different particular cases.

With the simple method described above it becomes very easy to decide if some given generalized effective action for gravity gives rise or not to exponential inflationary solutions and, in that case, to obtain an estimation of the change of the scale produced in the corresponding inflationary phase. In the next sections we will apply the method to two different scenarios which have been considered in the literature in different contexts.

\section{TWO-LOOP COUNTERTERM}

The first example we will consider is that of [5]. There the authors studied the minimal consistent effective low-energy two-loop renormalizable Lagrangian for pure gravity; this Lagrangian is shown to contain a six derivative term [6] added to the usual Einstein-Hilbert one:

$$
\mathcal{L}_{\mathrm{eff}}=-\frac{M_{P}^{2}}{16 \pi} R+\frac{\alpha}{M_{P}^{2}} R_{\delta \gamma}^{\alpha \beta} R_{\delta \rho}^{\sigma \gamma} R_{\alpha \beta}^{\sigma \rho} .
$$

The function $L(H, \dot{H})$ appearing in Eq. (9) is obtained just by rewriting Eq. (21) for the flat Robertson-Walker space:

$$
L(H, \dot{H})=\frac{6 M_{P}^{2}}{16 \pi}\left(\dot{H}+2 H^{2}\right)-24 \frac{\alpha}{M_{P}^{2}}\left[\left(\dot{H}+H^{2}\right)^{3}+H^{6}\right] .
$$

By solving Eq. (18) we find the inflationary periods produced as a consequence of the introduction of this higher order term. The equation reads

$$
\frac{18 M_{P}^{2}}{16 \pi} H_{0}^{2}+\frac{72 \alpha}{M_{P}^{2}} H_{0}^{6}=0
$$

with the obvious solutions $H_{0}=0$ and $H_{0}^{4}=$ $-M_{P}^{4} /(64 \pi \alpha)$. Therefore, there exists only one inflationary de Sitter period provided $\alpha$ is negative. On the other hand, we find one stable and one unstable mode for the perturbations to this de Sitter solution. Equation (20) gives the number of $e$ foldings produced before the universe leaves this state of exponential growing; $N_{e} \simeq 4.81$ is immediately obtained. It is important to notice that $N_{e}$ does not depend on the $\alpha$ coefficient preceding it. Therefore, the addition of the six derivative term to the Einstein-Hilbert action considered here does not seem to produce enough inflation to solve the problems of the standard cosmology.

\section{EFFECT OF MATTER}

This model is based on the semiclassical Einstein equations

$$
R_{\mu \nu}-\frac{1}{2} g_{\mu \nu} R=-8 \pi G\left\langle T_{\mu \nu}\right\rangle
$$

where $\left\langle T_{\mu \nu}\right\rangle$ is the vacuum expectation value of the stress tensor of a number of massless conformally invariant quantum fields with different spin values. As is well known this vacuum expectation value will be divergent in general and some regularization method will be needed to give it sense. These divergences affecting $\left\langle T_{\mu \nu}\right\rangle$ have been computed in the literature and cause the appearance of fourth order operators at the leading adiabatic order (see [18] for a very complete review). The corresponding higher order terms in the matter Lagrangian may be considered also as part of the gravitational effective Lagrangian since they are pure geometric objects, i.e., they depend only on the standard Riemann and Ricci tensor and the curvature scalar, but not on the matter fields themselves. In dimensional regularization and using the fact that $-4 R^{\alpha \beta}{ }_{\gamma \delta} R^{\gamma \delta}{ }_{\alpha \beta}+16 R^{\alpha \beta} R_{\alpha \beta}-4 R^{2}$ is a total divergence in four dimensions, this divergent contribution to the effective Lagrangian can be written for massless fields as

$$
\mathcal{L}_{\mathrm{div}}=\frac{1}{(4 \pi)^{2}}\left(\frac{1}{\epsilon}-\frac{\gamma_{e}}{2}\right) a_{2}(x)
$$

with

$$
\begin{gathered}
a_{2}(x)=\beta_{1} R^{2}+\gamma R_{\mu \nu} R^{\mu \nu} \\
\beta_{1}=-\frac{N_{\mathrm{sc}}}{180}+\frac{N_{\mathrm{gh}}}{90}+\frac{N_{\nu}}{60}+\frac{N_{\mathrm{fe}}}{30}-\frac{N_{v}}{20} \\
\gamma=\frac{N_{\mathrm{sc}}}{60}-\frac{N_{\nu}}{20}-\frac{N_{\mathrm{fe}}}{10}+\frac{7 N_{v}}{30}-\frac{N_{\mathrm{gh}}}{30}
\end{gathered}
$$

where $\gamma_{e}$ is the Euler constant and we have assumed $N_{\mathrm{sc}}$ scalars, $N_{\text {gh }}$ ghosts, $N_{\nu}$ neutrinos, $N_{\text {fe }}$ Dirac fermions, and $N_{v}$ vectors fields to be present.

Now we have to deal with the problem of the divergent coefficient multiplying these terms. In principle, one may estimate in a heuristic way the value of the corresponding renormalized coefficients by performing the substitution $1 / \epsilon-\gamma_{e} / 2 \rightarrow \ln \left(M_{P} / M\right)$ which is nearly equivalent to the assumption of integrating the matter fields modes from some infrared cutoff $M$ to the $M_{P}$ scale. Thus the corresponding renormalized effective Lagrangian will be 


$$
\mathcal{L}_{G}=-\frac{M_{P}^{2}}{16 \pi} R+\frac{1}{(4 \pi)^{2}} \ln \left(\frac{M_{P}}{M}\right) a_{2}(x) .
$$

On the other hand, the finite remainder $\left\langle T_{\mu \nu}\right\rangle_{\text {ren }}$ is in general quite difficult to compute. However, in the case of conformally flat spaces and quantum fields which are also conformally invariant, $\left\langle T_{\mu \nu}\right\rangle_{\text {ren }}$ can be exactly computed out of the knowledge of the trace anomaly and it renders

$$
\left\langle T_{\mu \nu}\right\rangle_{\text {ren }}=\beta_{2}^{(1)} H_{\mu \nu}+\rho^{(3)} H_{\mu \nu}+{ }^{(4)} H_{\mu \nu},
$$

where

$$
\begin{aligned}
& { }^{(1)} H_{\mu \nu}=2 R_{; \mu \nu}-2 g_{\mu \nu} \square R-\frac{1}{2} g_{\mu \nu} R^{2}+2 R R_{\mu \nu}, \quad(31) \\
& { }^{(3)} H_{\mu \nu}=R_{\mu}^{\sigma} R_{\nu \sigma}-\frac{2}{3} R R_{\mu \nu}-\frac{1}{2} g_{\mu \nu} R^{\sigma \tau} R_{\sigma \tau}+\frac{1}{4} g_{\mu \nu} R^{2}
\end{aligned}
$$

${ }^{(4)} H_{\mu \nu}$ is a border term and will be omitted. It is possible to derive the first term above ${ }^{(1)} H_{\mu \nu}$ from an action integral in this way:

$$
{ }^{(1)} H_{\mu \nu}=\frac{1}{\sqrt{-g}} \frac{\delta}{\delta g^{\mu \nu}} \int \sqrt{-g} R^{2} d^{n} x
$$

Note that in general ${ }^{(3)} H^{\mu \nu}$ cannot be obtained by varying a local action, although several nonlocal actions have been proposed which lead to this term. In spite of this, in the case we are considering, i.e., conformally flat space, a local action can be found [19] which can be written in terms of the Hubble parameter as

$$
\Gamma=\int d t e^{3 b} H^{4}
$$

It is then possible to write a matter Lagrangian in the case of conformally flat space which leads to $\left\langle T_{\mu \nu}\right\rangle_{\text {ren }}$ and in terms of the Hubble parameter takes the form

$$
L_{M}=\beta_{2}\left(\dot{H}+2 H^{2}\right)^{2}+\rho H^{4},
$$

with the coefficients $\beta_{2}$ and $\rho$ given by

$$
\beta_{2}=-\frac{1}{144 \pi^{2}} \frac{1}{120}\left(N_{\mathrm{sc}}+3 N_{\nu}+6 N_{\mathrm{fe}}-18 N_{v}-2 N_{\mathrm{gh}}\right),
$$

$\rho=-\frac{1}{8 \pi^{2}} \frac{1}{360}\left(-N_{\mathrm{sc}}-\frac{11}{2} N_{\nu}-11 N_{\mathrm{fe}}-62 N_{v}+2 N_{\mathrm{gh}}\right)$.

Therefore we can write the function $L(H, \dot{H})$ in Eq. (9) including the gravitational and matter sectors as

$$
\begin{aligned}
L(H, \dot{H})= & L_{G}+L_{M}=\frac{6 M_{P}^{2}}{16 \pi}\left(\dot{H}+2 H^{2}\right)+\rho H^{4} \\
& +36\left[\frac{1}{(4 \pi)^{2}} \ln \left(\frac{M_{P}}{M}\right) \beta_{1}+\beta_{2}\right]\left(\dot{H}+2 H^{2}\right)^{2} \\
& +12 \frac{1}{(4 \pi)^{2}} \ln \left(\frac{M_{P}}{M}\right) \gamma\left(\dot{H}^{2}+3 \dot{H} H^{2}+3 H^{4}\right) .
\end{aligned}
$$

As we did in the previous example we use Eq. (18) in order to get the inflationary periods and we obtain, in this case,

$$
-\frac{M_{P}^{2}}{8 \pi} H_{0}^{2}+\rho H_{0}^{4}=0
$$

whose solutions are simply $H_{0}=0$ and $H_{0}^{2}=M_{P}^{2} /(8 \pi \rho)$. Thus we find again only one de Sitter phase provided $\rho$ is positive. Finally Eq. (20) will provide the number of $e$ folds during this phase. For an infrared cutoff $M \simeq 100$ GeV Eq. (20) yields

$$
\frac{1}{N_{e}}=-\frac{3}{2}+\sqrt{\frac{9}{4}+\frac{6 \rho}{8.92 \beta_{1}+2.97 \gamma+36 \beta_{2}}} .
$$

One interesting case to apply the above results to is the standard model of elementary particles interactions based on the gauge group $\mathrm{SU}(3)_{C} \times \mathrm{SU}(2)_{L} \times \mathrm{U}(1)_{Y}$; we have $N_{\mathrm{sc}}=4, N_{\nu}=3, N_{\mathrm{fe}}=21, N_{v}=12$, and $N_{\mathrm{gh}}=12$. By substituting for these values in Eqs. (39) and (40), one obtains $H_{0}=1.08 M_{P}$ and $N_{e} \simeq 44$. Note that the material content of the standard model is not strictly speaking conformal matter, since many dimensional parameters appear in the corresponding Lagrangian. However, at the high energies which we are interested in, these parameters can be neglected and all the standard model particles can be considered as massless. In addition we are also ignoring the effect of the gauge interactions but still this could be a not so bad approximation at higher energies because of the asymptotic freedom property of the strong interactions. In a similar fashion, other models with additional matter content can also be considered.

So far we have considered only the possibility of having de Sitter inflation; now we will study the general case. To determine the set of initial conditions required to have a period of inflation we need to study the specific phase space of this model. The equations of motion for $H$ [Eq. (10)] corresponding to the Lagrangian of this model [Eq. (38)] are third order ordinary differential equations. However, since the time variable does not appear explicitly in the action we can reduce the degree of the equation by finding a constant of motion. This constant is given by

$$
\begin{aligned}
e^{3 b}\left(\dot{H} \frac{\partial L}{\partial \dot{H}}-3 H^{2} \frac{\partial L}{\partial \dot{H}}-H \frac{d}{d t} \frac{\partial L}{\partial \dot{H}}+H \frac{\partial L}{\partial H}\right. & -L) \\
& =\text { const. }
\end{aligned}
$$

Consequently the equations of motion for $H$ obtained from any generalized action (provided it is a scalar function on the Riemann and Ricci tensors and the scalar curvature but not on their derivatives) can always be written as a second order autonomous equation.

Taking the value of the constant equals zero we find the following second order equation for the standard model parameters:

$$
\ddot{H}=\frac{3 \dot{H}^{2}-18 \dot{H} H^{2}-0.12 H^{2}+0.10 H^{4}}{6 H},
$$




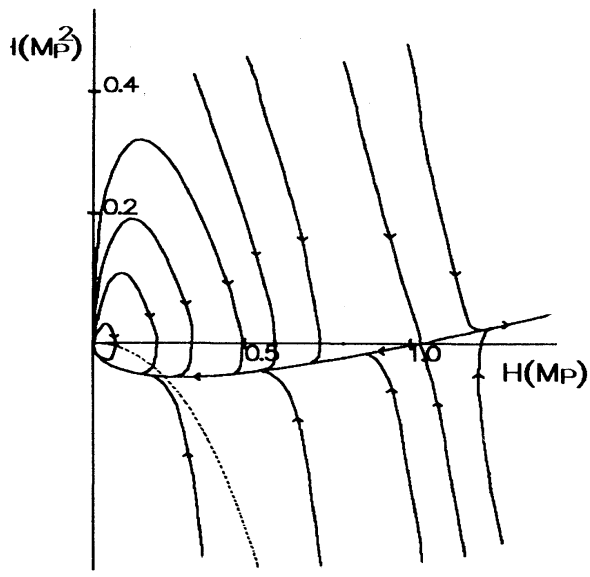

FIG. 1. Qualitative picture of the phase map corresponding to Eq. (42). The dotted line corresponds to the curve $\dot{H}=-H^{2}$.

where we have taken $M_{P}=1$. The corresponding phase map is shown in Fig. 1. We first note the de Sitter solution corresponding to $H=1.08$ and $\dot{H}=0$. The dotted line corresponds to the curve $\dot{H}=-H^{2}$ which separates the inflationary region from the noninflationary one as we discussed above. We see that there exists a large region of initial conditions which gives rise to inflation. On the other hand, we must mention that at late times any solution either grows indefinitely, as it occurs with those placed to the right of the de Sitter solution, or falls into the Minkowski solution allowing, at least in principle, a matching with the standard Friedmann-RobertsonWalker cosmology. Near the origin, for $H \ll M_{P}$ and $\dot{H} \ll M_{P}^{2}$, Eq. (42) reduces to

$$
\ddot{H}=\frac{3 \dot{H}^{2}-0.12 H^{2}}{6 H} .
$$

Its phase map is represented in Fig. 2. This equation possesses the exact solution

$$
H(t)=H\left(t_{0}\right) \cos ^{2}\left(-\sqrt{\frac{M_{P}^{2}}{32 \pi}}\left(t-t_{0}\right)\right) .
$$

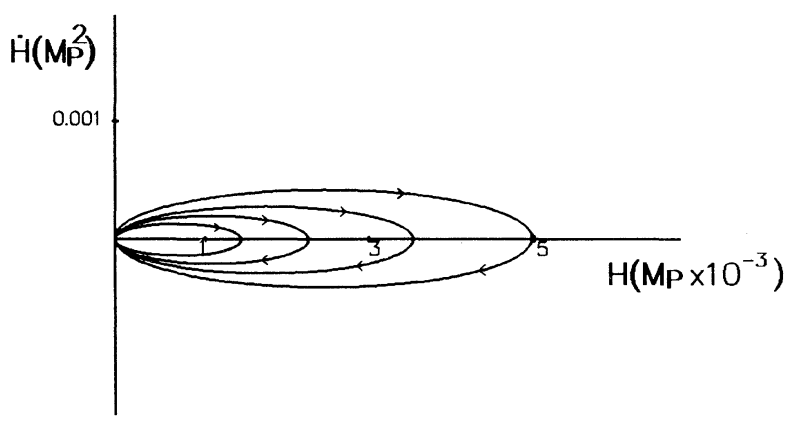

FIG. 2. Qualitative picture of the phase map corresponding to Eq. (43).
However, this solution is valid only for values $M \ll$ $H \ll M_{P}$ where $M$ is the infrared cutoff since the approximations we have done in the above computations break down at low energies. In particular, for energies lower than $M$ the matter fields degrees of freedom should appear explicitly modifying the evolution equations and particle masses and gauge interactions cannot be neglected any more.

In any case, the numerical results obtained can be used to make an estimation of the number of foldings [as defined in Eq. (20] for general initial conditions. Of course this number depends on these initial conditions. However, in order to have an idea of the typical orders of magnitude, we have considered some examples. For instance, for $H=1$ and $\dot{H}=0$ we obtain $N_{e} \simeq 135$ and for $H=0.5$ and $\dot{H}=0, N_{e} \simeq 22$. Thus, by looking at Fig. 1, one can conclude that there exists a wide region of initial conditions which give rise to a large expansion factor inside the inflationary region $\dot{H}>-H^{2}$.

Apart from having a large enough number of foldings one should also find a successful exit into the standard hot big-bang model. As discussed above, the corresponding detailed analysis cannot be done in the framework of the simplified model studied here since it applies only to energies larger than the infrared cutoff $M$. Moreover, a detailed study of a generalization of the model considered above done in [20] showed that, for the values of the parameters that describe the standard model matter, the fluctuations in the conformal background gravitational field give rise to density perturbations which are too large. However, this study does not include explicitly the effect of matter fields and it is not completely clear if its conclusions apply to the case of the standard model matter coupled to classical gravity. In particular the conformal invariance assumed in the analysis done in [20] is lost at low energies due to the masses and the running of the couplings discussed above.

\section{CONCLUSIONS}

In this work we have studied the possibility of having a phase of de Sitter inflation starting from an effective Lagrangian for gravity which is an arbitrary function of the Riemann and Ricci tensors and the curvature scalar. Therefore, our main assumption is that there exists some epoch in the early history of our universe where its evolution can be described in a classical way but with an unknown effective action for the gravitational field. We have set the precise conditions on this action for such an exponential expansion to take place. We have shown that it is a rather common phenomenon in four dimensions, even without adding a cosmological constant, since for a generic action Eq. (5) may have solutions different from zero.

We have also studied the stability of the de Sitter solution and estimate its duration in classical terms. With these formal tools we have considered two particular models giving rise to higher derivative terms for the gravity action. 
The first case to which we have applied our general method is the study of the effect of including the six derivative term needed to renormalize two-loop pure quantum gravity. With this new term added to the standard Einstein-Hilbert action we find that an exponential de Sitter solution appears with one unstable mode. The corresponding inflationary phase produces 4.8 foldings independently of the concrete value of the six derivative term coupling. Thus we can conclude that this new term does not seem to produce inflation enough to solve any of the problems of the standard cosmology.

As a second example we have applied our general method to study the possibility of having inflation driven by the terms induced in the effective low-energy action for gravitation by integrating out the matter fields above some cutoff $M$. In particular we have found the interesting result that the standard model matter produces, by itself, an inflationary de Sitter phase with $N_{e} \simeq 44$. Moreover, we have studied in detail the phase space of this model and we concluded that there is a wide region of possible initial conditions (outside the pure de Sitter solution) which also gives rise to an important number of foldings, defined according to Eq. (20), and with $\dot{H}>-H^{2}$ in most of the time of the correspond- ing evolution. This last remark is important concerning the possible solution of the horizon problem. Nevertheless, from the work in [20], we know that this model could produce too large density fluctuations if conformal invariance is assumed to be a good approximation to describe the standard model coupled to gravity even at energies below the infrared cutoff although this is not certainly the case.

In conclusion we consider that, in absence of a fundamental theory of gravitation, the possibility of having inflation without the somewhat artificial artifact of the inflaton field should not be ruled out. Moreover, the results found here for the case of the standard model matter coupled to classical gravity seem to suggest that it is worth studying in depth the dynamics of this system that, finally, is the only one that we are sure is realized in nature. Work is in progress in this direction.

\section{ACKNOWLEDGMENTS}

This work has been partially supported by the Ministerio de Educación y Ciencia (Spain) (CICYT AEN930776).
[1] A. H. Guth, Phys. Rev. D 23, 347 (1981).

[2] A. D. Linde, Rep. Prog. Phys. 47, 925 (1984).

[3] A. A. Starobinsky, Phys. Lett. 91B, 99 (1980).

[4] A. Vilenkin, Phys. Rev. D 32, 2511 (1985).

[5] A. Dobado and A. López, Phys. Lett. B 316, 250 (1993).

[6] D. M. Capper, J. J. Dulwich, and M. Ramón Medrano, Nucl. Phys. B254, 737 (1985); M. H. Goroff and A. Sagnotti, ibid. B266, 709 (1986).

[7] S. Weinberg, Physica A 96, 327 (1979).

[8] J. Gasser and H. Leutwyler, Ann. Phys. (N.Y.) 158, 142 (1984); Nucl. Phys. B250, 465 (1985); B250, 517 (1985).

[9] A. Dobado and M. J. Herrero, Phys. Lett. B 228, 495 (1989); 233, 505 (1989); J. Donoghue and C. Ramirez, ibid. 234, 361 (1990).

[10] J. F. Donoghue, Phys. Rev. Lett. 72, 2996 (1994).

[11] I. L. Buchbinder, S. D. Odintsov, and I. L. Shapiro, Effective Action in Quantum Gravity (IOP, Bristol, 1992).

[12] J. Z. Simon, Phys. Rev. D 43, 3308 (1991).

[13] T. H. R. Skyrme, Proc. R. Soc. London 260, 127 (1961);
T. H. R. Skyrme, Nucl. Phys. 31, 556 (1962); G. S. Adkins, C. R. Nappi, and E. Witten, Nucl. Phys. B228, 552 (1983).

[14] J. D. Barrow and A. C. Ottewill, J. Phys. A 16, 2757 (1983).

[15] B. Whitt, Phys. Lett. 145B, 176 (1984); K. Maeda, Phys. Rev. D 39, 3159 (1989); D. Wands, Class. Quantum Grav. 11, 269 (1994).

[16] G. Magnano, M. Ferraris, and M. Francaviglia, Class. Quantum Grav. 7, 557 (1990).

[17] S. Weinberg, Gravitation and Cosmology (Wiley, New York, 1972).

[18] N. D. Birrell and P. C. W. Davies, Quantum Fields in Curved Space (Cambridge University Press, Cambridge, England, 1982).

[19] M. V. Fischetti, J. B. Hartle, and B. L. Hu, Phys. Rev. D 20, 1757 (1979).

[20] L. A. Kofman, V. F. Mukhanov, and D. Yu. Pogosyan, Sov. Phys. JETP 66, 433 (1987). 